EDITORIAL

\title{
Diastolic heart failure: fact or fiction?
}

\section{J E Sanderson}

Heart 2003;89:1281-1282

It is logical to find some abnormalities of systolic function in those with "diastolic" heart failure, just as it is well established that diastolic dysfunction is an integral part of systolic heart failure

$\mathrm{T}$ he concept of "diastolic" heart failure grew out of the observation that many patients who had the symptoms and signs of heart failure had an apparently normal left ventricular $(\mathrm{LV})$ ejection fraction $(>0.45)$. Thus it was assumed that since systolic function was "preserved" the problem must lie in diastole, although it is not clear by whom or when this assumption was made. Nevertheless, many guidelines followed on how to diagnose "diastolic" heart failure backed up by indicators of diastolic dysfunction derived from Doppler echocardiography, ${ }^{1-3}$ and it was found to be common. ${ }^{4}$ This edifice is, however, built on a number of assumptions that are looking increasingly shaky. For example, is the ejection fraction a good index of LV systolic function? Can diastolic function or dysfunction be measured accurately? And in any case can systole be separated so neatly from diastole?

\section{EJECTION FRACTION}

The ejection fraction has proved to be a robust measurement for epidemiological purposes but in individuals it is highly unsatisfactory-it is dependent on many variables including most importantly afterload. Furthermore it tends to reflect mainly circumferential fibre shortening and takes little account of long axis function. In fact, studies of long axis shortening using the mitral valve ring have shown that maximal systolic excursion by $\mathrm{M}$ mode echocardiography or peak systolic velocity by tissue Doppler imaging are remarkably sensitive indicators of systolic performance. ${ }^{5}$ We, ${ }^{6}$ and others, ${ }^{7}$ have used these techniques in patients with diastolic heart failure diagnosed according to the European guidelines, and shown conclusively that mean systolic mitral annular amplitude and peak velocity were significantly lower than normal age matched controls, although higher than those with "systolic" heart failure. ${ }^{6}$ Recently end systolic elastance (stiffness) was also shown to be higher in patients with heart failure with "preserved ejection fraction". ${ }^{8}$ Thus, if a sensitive enough tool is used it can be shown that systolic function is not normal in those with diastolic heart failure despite a so-called normal LV ejection fraction. The majority (95\%) of our patients had LV hypertrophy suggesting that most of these patients with diastolic heart failure probably have hypertensive heart failure. Frequently the heart failure is precipitated either by some degree of ischaemia or an arrhythmia.

The question of whether we have the tools or the concepts to adequately describe and define diastolic function or its abnormalities has recently been lucidly covered in this journal by Gibson and Francis." As these authors state "no simple definition of diastolic disease has emerged". Notwithstanding the lack of a theoretical framework, standard Doppler echocardiography indices derived from mitral inflow have been widely used for a crude or rough assessment of diastolic function. Although a restrictive filling pattern is clearly abnormal and related to high atrial pressure and natriuretic peptide concentrations, ${ }^{10}$ the so called abnormal filling pattern is strongly affected by heart rate and age, and is virtually universal in elderly subjects. ${ }^{11}$ Of course, age related changes are not normal and increasing ventricular stiffness is part of the aging process. However, the presence of an abnormal relaxation pattern cannot be taken as more abnormal than "normal" aging. Even if pressure-volume loops are derived at cardiac catheterisation, the measurement of a ventricular stiffness constant by applying an exponential equation is not measuring stiffness but the extent that stiffness varies with volume changes, as pointed out by Gibson and Francis. ${ }^{9}$ Even a perfectly normal heart will become abnormally stiff if the volume is increased enough. Therefore, there is no gold standard for diastolic dysfunction and we do not have the tools to accurately measure diastolic function; however, the early diastolic velocity of the mitral annulus is a very sensitive measure of impaired overall ventricular function, indicating the value of assessing ventricular long axis function. ${ }^{12}$

\section{SYSTOLIC FUNCTION}

It is often overlooked that the major determinant of diastolic filling is systolic function. Incoordination during contraction leads to a notable delay in mitral opening and curtailment of the time for filling, and impaired contractility will reduce ventricular suction. This latter process is vital for rapid early ventricular filling and is likely to be the first process affected by declining systolic function. In fact, it is theoretical nonsense to try to artificially separate systole from diastole-they are both part of a single contraction-relaxation cycle and a continuous process. ${ }^{13}{ }^{14}$ It is likely and logical therefore to expect to find some abnormalities of systolic function in those with "diastolic" heart failure, just as it is well established that diastolic dysfunction is an integral part of systolic heart

failure. ${ }^{11}$ In fact, the diastolic abnormalities in 
those with "systolic" heart failure are more powerful predictors of outcome and mortality than the systolic abnormalities..$^{15} 16$

It is time therefore to abandon this artificial and increasingly meaningless distinction between systolic and diastolic heart failure. This taxonomy has run its course and is no longer useful. Patients present with what appears to be heart failure clinically and it is better to try to determine the precise cause and treat appropriately. Each patient will have a mixture of systolic and diastolic abnormalities with some degree of incoordination. Most with so-called "diastolic" heart failure will be elderly ladies who have hypertensive heart failure in disguise, and those with "systolic" heart failure will have severe ischaemic heart disease or cardiomyopathy with pronounced ventricular remodelling. The consequence of using the LV ejection fraction in the past to classify patients has meant that almost half of our heart failure patients have been excluded from all the previous heart failure treatment trials, and these studies will now have to be done again in a more representative cohort. As throughout history, plausible but incorrect theories can have dire practical consequences.

\section{REFERENCES}

1 European Study Group on Diastolic Heart Failure. European study group on diastolic heart failure working group report: how to diagnose diastolic heart failure. Eur Heart J 1998; 19:990-1003.

2 Vasan RS, Levy D. Defining diastolic heart failure: a call for diagnostic criteria. Circulation 2000;101:2118-21.
3 Zile MR, Brutsaert DL. New concepts in diastolic dysfunction and diastolic heart failure: part I: diagnosis, prognosis, and measurement of diastolic function. Circulation 2002;105:1387-93.

4 Yip GWK, Ho PPY, Woo KS, et al. Comparison of frequencies of left ventricular systolic and diastolic heart failure in Chinese living in Hong Kong. Am J Cardiol 1999;84:563-7.

5 Henein MY, Gibson DG. Long axis function in disease. Heart 1999;81:229-31.

6 Yip GWK, Wang $M$, Zhang $Y$, et al. Left ventricular long axis function in diastolic heart failure is reduced in both diastole and systole: time for a redefinition. Heart 2002;87:121-5.

7 Petrie MC, Caruana L, Berry C, et al. "Diastolic heart failure or heart failure caused by subtle left ventricular systolic dysfunction. Heart 2002;87:29-31.

8 Kawaguchi MH, Fetics B, Kass DA. Combined ventricular systolic and arterial stiffening in patients with heart failure and preserved ejection fraction: implications for systolic and diastolic reserve limitations. Circulation 2003; 107:714-20.

9 Gibson DG, Francis DP. Clinical assessment of left ventricular diastolic function. Heart 2003:89:231-8.

10 Yu CM, Sanderson JE, Shum IO, et al. Diastolic dysfunction and natriuretic peptides in systolic heart failure. Hlgher ANP and BNP levels are associated with the restrictive filling pattern. Eur Heart J 1996;17:1694-702.

11 Yu CM, Sanderson JE. Right and left ventricular diastolic function in patients with and without heart failure: effect of age, sex, heart rate, and respiration on Doppler-derived measurements. Am Heart J 1997; 134:426-34.

12 Wang M, Yip GW, Wang AY, et al. Peak early diastolic mitral annulus velocity by tissue Doppler imaging adds independent and incremental prognostic value. J Am Coll Cardiol 2003:41:820-6.

13 Brutsaert DL, Sys SU. Relaxation and diastole of the heart. Physiol Rev 1989;69:1228-315.

14 Yip GWK, Zhang Y, Tan PY, et al. Left ventricular long-axis changes in early diastole and systole: impact of systolic function on diastole. Clin Sci (Lond) 2002;102:515-22.

15 Xie GY, Berk MR, Smith MD. Prognostic value of Doppler transmitral flow patterns in patients with congestive heart failure. J Am Coll Cardiol 1994:24:132-9.

16 Yu HCM, Sanderson JE. Different prognostic significance of right and left ventricular diastolic dysfunction in heart failure. Clin Cardiol 1999;5:117-26.

\section{IMAGES IN CARDIOLOGY}

\section{Large B cell lymphoma with cardiac infiltration}

A

22 year old woman was referred to our institution with shortness of breath and palpitations that had worsened over the preceding two weeks. She had also lost $10 \mathrm{~kg}$ in weight in the preceding eight months. On chest $x$ ray, a large mediastinal mass was found. Transthoracic echocardiography revealed a large tumour infiltrating both atria, including a $20 \times 20 \mathrm{~mm}$ growth in the right atria (white arrows, left panel) and mild pericardial effusion. The diagnosis of a diffuse large $B$ cell lymphoma was reached following transbronchial biopsy. The patient underwent two cycles of CHOP chemotherapy (cyclophosphamide 750 $\mathrm{mg} / \mathrm{m}^{2}$, doxorubicin $50 \mathrm{mg} / \mathrm{m}^{2}$, vincristine $2 \mathrm{mg}$, and prednisone) within 14 days. Echocardiographic follow up 12 days after the second chemotherapy cycle showed complete remission of the large atrial mass of the right atria and both atrial walls (right panel). No pulmonary or cerebral embolism occurred, and no atrial septal defect or pericardial effusion were seen after chemotherapy.
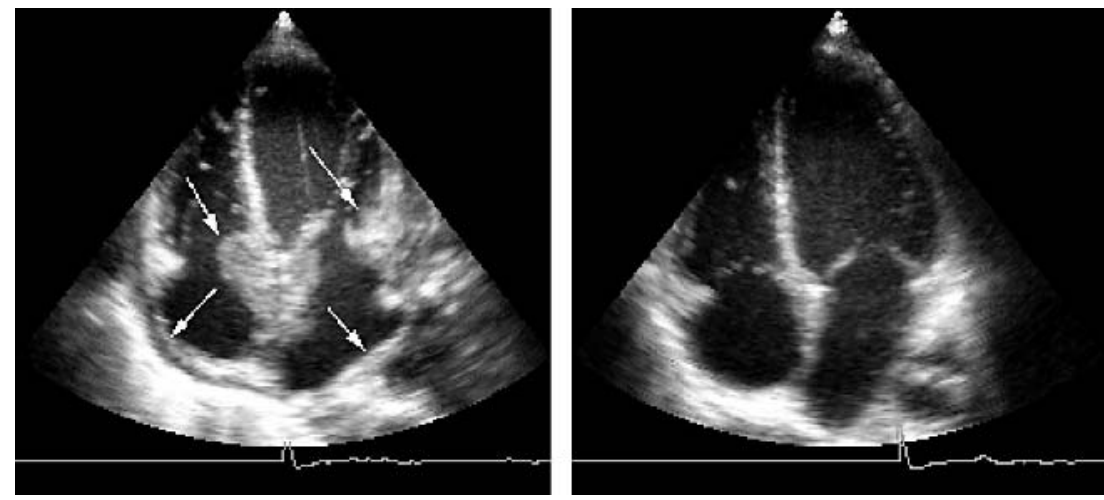

F Koehler

A C Borges

P C Fotuhi

parwis.fotuhi@charite.de 